\title{
LIMIT LAWS FOR SUMS OF INDEPENDENT RANDOM PRODUCTS: THE LATTICE CASE
}

\author{
ZAKHAR KABLUCHKO
}

\begin{abstract}
Let $\left\{V_{i, j} ;(i, j) \in \mathbb{N}^{2}\right\}$ be a two-dimensional array of i.i.d. random variables. The limit laws of the sum of independent random products$$
Z_{n}=\sum_{i=1}^{N_{n}} \prod_{j=1}^{n} e^{V_{i, j}}
$$

as $n, N_{n} \rightarrow \infty$ have been investigated by a number of authors. Depending on the growth rate of $N_{n}$, the random variable $Z_{n}$ obeys a central limit theorem, or has limiting $\alpha$-stable distribution. The latter result is true for non-lattice $V_{i, j}$ only. Our aim is to study the lattice case. We prove that although the (suitably normalized) sequence $Z_{n}$ fails to converge in distribution, it is relatively compact in the weak topology, and describe its cluster set. This set is a topological circle consisting of semi-stable distributions.
\end{abstract}

\section{INTRODUCTION AND STATEMENT OF RESUlts}

Let $\left\{V_{i, j} ;(i, j) \in \mathbb{N}^{2}\right\}$ be a two-dimensional array of independent copies of a realvalued random variable $V$. Our main object of interest is the sum of independent random products

$$
Z_{n}=\sum_{i=1}^{N_{n}} \prod_{j=1}^{n} e^{V_{i, j}} .
$$

Here, $N_{n}$ is a sequence of positive integers converging to $\infty$. The limit laws of the random variable $Z_{n}$ as $n, N_{n} \rightarrow \infty$ have been studied by Bovier et al. [4] for Gaussian $V$ (see Theorems 1.5, 1.6 therein), and by Cranston and Molchanov [5] for arbitrary $V$ with finite exponential moments. The study of $Z_{n}$ is motivated by a number of models in statistical physics. To mention only one example, if $V_{i, j}$ are Gaussian variables, then $Z_{n}$ is the partition function of the random energy model; see [4]. The character of the limiting distribution of $Z_{n}$ depends on the growth rate of the sequence $N_{n}$. If the sequence $N_{n}$ grows fast in the sense that

$$
\liminf _{n \rightarrow \infty} \frac{1}{n} \log N_{n}>\lambda_{2}
$$

for some critical value $\lambda_{2}>0$ depending only on the distribution of $V$, then the random variable $Z_{n}$ obeys a central limit theorem with the usual normalization:

$$
\frac{Z_{n}-\mathbb{E} Z_{n}}{\sqrt{\operatorname{Var} Z_{n}}} \stackrel{\mathrm{w}}{\rightarrow} \mathcal{N}(0,1), \quad n \rightarrow \infty .
$$

2000 Mathematics Subject Classification. Primary, 60G50; Secondary, 60F05, 60F10.

Key words and phrases. Random products, random exponentials, semi-stable laws, random energy model, triangular arrays, central limit theorem. 
If the sequence $N_{n}$ grows slowly in the sense that

$$
\lambda:=\lim _{n \rightarrow \infty} \frac{1}{n} \log N_{n} \in\left(0, \lambda_{2}\right),
$$

then the central limit theorem breaks down. Instead, for suitable normalizing sequences $A_{n}, B_{n}$, we have

$$
\frac{Z_{n}-A_{n}}{B_{n}} \stackrel{\mathrm{w}}{\rightarrow} \mathcal{F}_{\alpha}, \quad n \rightarrow \infty,
$$

where $\mathcal{F}_{\alpha}$ is an $\alpha$-stable distribution totally skewed to the right, and the stability parameter $\alpha \in(0,2)$ depends on $\lambda$. The proofs of (3) and (5) can be found in [5]. Unaware of [5], the author proved essentially the same results in [10]. A functional version of these results can be found in [11]. There is also a transition between the two regimes (2) and (4) taking place at $\log N_{n} \approx \lambda_{2} n$; see [4, Thm. 1.5(ii)], [5, Thm. 1.2], as well as [11, Thm. 1.3].

In their proof of the stable limit law (5), Cranston and Molchanov [5] relied on an asymptotic expansion in the central limit theorem; see Theorem 1 on page 210 in [8]. It has been overlooked in [5] that this result is true for non-lattice distributions only; see page 212 in [8] for a discussion of this fact. Recall that a random variable $V$ is called lattice if there exist $h, a \in \mathbb{R}$ such that the values of $V$ are a.s. of the form $h n+a, n \in \mathbb{Z}$.

Our aim is to investigate the lattice case. On the one hand, we will see that in this case the convergence to an $\alpha$-stable law breaks down. More precisely, there is no affine normalization which makes the sequence of random variables $Z_{n}$ weakly convergent. On the other hand, we will prove that for suitable $A_{n}$ and $B_{n}$, the sequence of random variables $\left(Z_{n}-A_{n}\right) / B_{n}$ is relatively compact in the weak topology and describe the set of weak accumulation points for this sequence. This set is a topological circle consisting of semi-stable distributions.

Let us state our results precisely. Let $V$ be a non-degenerate random variable satisfying the Cramér condition

$$
\psi(t):=\log \mathbb{E} e^{t V}<+\infty \text { for all } t \in \mathbb{R} .
$$

Let $I: \mathbb{R} \rightarrow[0,+\infty]$ be the Legendre-Fenchel transform of $\psi$ given by

$$
I(\beta):=\sup _{t \in \mathbb{R}}(\beta t-\psi(t)), \quad \beta \in \mathbb{R} .
$$

Following [5], define the "critical points" $\lambda_{1}$ and $\lambda_{2}, 0<\lambda_{1}<\lambda_{2}$, by

$$
\lambda_{1}:=\psi^{\prime}(1)-\psi(1), \quad \lambda_{2}:=2 \psi^{\prime}(2)-\psi(2) .
$$

We assume that the distribution of the random variable $V$ is lattice. Since $Z_{n}$ changes only by a constant factor $e^{-n a}$ if we replace $V$ by $V-a$, there is no restriction of generality in making the following assumption.

Assumption 1. There is $h>0$ such that the values of $V$ belong with probability 1 to the lattice $h \mathbb{Z}=\{h n ; n \in \mathbb{Z}\}$, and, moreover, $h$ is the largest number with this property.

It will be convenient to denote by $[b]_{h}$ and $\{b\}_{h}$ the entire part and the fractional part of $b \in \mathbb{R}$ taken with respect to the lattice $h \mathbb{Z}$, i.e.

$$
[b]_{h}:=\max \{a \in h \mathbb{Z}: a \leq b\}, \quad\{b\}_{h}:=b-[b]_{h} \in[0, h) .
$$

Note that $[b]_{1}$ and $\{b\}_{1}$ are the usual integer and fractional parts of $b$. The next theorem is our main result. 
Theorem 1. Suppose that (4), (6) and Assumption [1 are satisfied. Define $\alpha \in$ $(0,2)$ as the unique solution of the equation $\alpha \psi^{\prime}(\alpha)-\psi(\alpha)=\lambda$. Define $A_{n}$ and $B_{n}$ by

$$
\begin{aligned}
& A_{n}= \begin{cases}0, & \text { if } \lambda \in\left(0, \lambda_{1}\right), \\
N_{n} \mathbb{E}\left[e^{\sum_{j=1}^{n} V_{1, j}} 1_{\sum_{j=1}^{n} V_{1, j}<b_{n}}\right], & \text { if } \lambda=\lambda_{1}, \\
\mathbb{E} Z_{n}, & \text { if } \lambda \in\left(\lambda_{1}, \lambda_{2}\right),\end{cases} \\
& B_{n}=e^{b_{n}} \text {, where } b_{n}=n I^{-1}\left(\frac{1}{n} \log \left(\frac{N_{n} h}{\sqrt{2 \pi \psi^{\prime \prime}(\alpha) n}}\right)\right) \text {. }
\end{aligned}
$$

If $\left\{n_{k}\right\}_{k \in \mathbb{N}}$ is an increasing integer sequence such that

$$
\Delta:=\lim _{k \rightarrow \infty}\left\{b_{n_{k}}\right\}_{h} \in[0, h],
$$

then we have the following weak convergence:

$$
\frac{Z_{n_{k}}-A_{n_{k}}}{B_{n_{k}}} \stackrel{\mathrm{w}}{\rightarrow} \mathcal{F}_{\alpha, \Delta}, \quad k \rightarrow \infty .
$$

Here, $\mathcal{F}_{\alpha, \Delta}$ is an infinitely divisible distribution whose characteristic function $\phi_{\alpha, \Delta}$ has a Lévy-Khintchine representation

$$
\log \phi_{\alpha, \Delta}(u)=i C_{\alpha, \Delta ; \tau} u+\sum_{x \in e^{h \mathbb{Z}-\Delta}}\left(e^{i u x}-1-i u x 1_{x<\tau}\right) x^{-\alpha}, \quad u \in \mathbb{R},
$$

where $e^{h \mathbb{Z}-\Delta}$ denotes the geometric progression $\left\{e^{h n-\Delta} ; n \in \mathbb{Z}\right\}, \tau>0$ is arbitrary such that $\tau \notin e^{h \mathbb{Z}-\Delta}$, and $C_{\alpha, \Delta ; \tau}$ is a constant.

Corollary 1. Under the assumptions of Theorem 1, the sequence of random variables

$$
\left\{\frac{Z_{n}-A_{n}}{B_{n}} ; n \in \mathbb{N}\right\}
$$

is relatively compact in the weak topology. The set of the weak accumulation points of the sequence (14) is $\left\{\mathcal{F}_{\alpha, \Delta} ; \Delta \in[0, h]\right\}$. Endowed with the induced weak topology, this set is homeomorphic to a circle.

Example 1. Let the variable $V$ take two values $h$ and 0 with probabilities $p$ and $1-p$, respectively, $p \in(0,1)$. In order to motivate this choice, consider a game in which a player with starting capital 1 tosses a coin $n$ times and each time the coin shows heads (which happens with probability $p$ ), the capital is multiplied by $e^{h}$. If the coin lands tails, the capital remains unchanged. With other words, the gain of the player in such a game is $e^{h k}$ if the coin lands $k$ times heads, $k=0, \ldots, n$. Then, the random variable $Z_{n}$ may be interpreted as the total gain in $N_{n}$ independent games. Theorem 1 provides a complete description of the subsequential limit laws of $Z_{n}$ as $n, N_{n} \rightarrow \infty$ provided that the growth condition (44) is satisfied. The critical point $\lambda_{2}$ is given by

$$
\lambda_{2}=\frac{2 p h e^{2 h}}{(1-p)+p e^{2 h}}-\log \left((1-p)+p e^{2 h}\right) .
$$

It should be stressed that the central limit theorem (3) as well as the limit results in the intermediate regime $\log N_{n} \approx \lambda_{2} n$ (see [11, Thm. 1.3]) remain valid in the lattice case. Also, the strong laws for $Z_{n}$ proved in [12], [5], [10] hold in the lattice 
case. Thus, it is only the weak convergence result under the growth condition (4) which is affected by the lattice assumption.

Remark 1. The distributions $\mathcal{F}_{\alpha, \Delta}$ are semi-stable. Recall that an infinitely divisible distribution on the real line with characteristic function $\phi$ is called semi-stable with index $\alpha \in(0,2]$ if for some positive $a \neq 1$, there exists $c \in \mathbb{R}$ such that $(\phi(u))^{a}=e^{i c t} \phi\left(a^{1 / \alpha} u\right)$; see [18, Ch. 3]. Stable distributions are obtained by requiring the same condition to hold for every $a>0$ (with $c$ depending on $a$ ). Semi-stable distributions arise as subsequential weak limits of the partial sums of i.i.d. random variables taken along geometrically growing subsequences; see, e.g., 17]. This setting is applicable for example to the total gain in a large number of St. Petersburg games. Recall that in a St. Petersburg game, the gain of a player is $2^{k}$ with probability $2^{-k}, k \in \mathbb{N}$. If $S_{N}$ denotes the total gain in $N$ independent St. Petersburg games, then the random variable $S_{N}$ does not converge to a limiting distribution as $N \rightarrow \infty$. However, it has been observed by Martin-Löf [15] that the subsequence $S_{2^{N}}$ has a limiting distribution as $N \rightarrow \infty$. Later, the full picture of limiting semistable laws arising as subsequential limits of $S_{n}$ has been established in [6]. It is interesting to note that although the sum of independent products $Z_{n}$ does not fit in this setting (rather, $Z_{n}$ is a row sum in a triangular array), the structure of the set of limiting distributions is very similar to that encountered in [6]. In particular, the limiting distributions are semi-stable (and not only infinitely divisible, which is clear a priori).

Remark 2. In (10), we agree to take the values of the inverse function $I^{-1}$ to be in the interval $\left(\beta_{0}, \beta_{+\infty}\right)$. Note that by (44), see also Eqn. (24) below, we have $b_{n} \sim \psi^{\prime}(\alpha) n$ as $n \rightarrow \infty$.

Remark 3. The value of the constant $C_{\alpha, \Delta ; \tau}$ is given by Eqns. (48), (52), (56) below for $\alpha \in(0,1), \alpha=1, \alpha \in(1,2)$, respectively. It is easily seen from these equations that the right-hand side of (13) does not depend on the choice of $\tau$.

Remark 4. Our growth condition (4) is less restrictive than the corresponding assumption in [5], where $N_{n}$ is chosen to be of the form $N_{n}=\alpha\left(2 \pi \psi^{\prime \prime}(\alpha) n\right)^{1 / 2} e^{\lambda n}$. See [11, Theorem 1.4] for the proof of (5) in the non-lattice case under (4).

A quantity closely related to the sum of independent products $Z_{n}$ is the maximum of independent products

$$
M_{n}=\max _{i=1, \ldots, N_{n}} \prod_{j=1}^{n} e^{V_{i, j}} .
$$

Equivalently, one may consider the maximum of independent sums

$$
\log M_{n}=\max _{i=1, \ldots, N_{n}} \sum_{j=1}^{n} V_{i, j} .
$$

The limiting behavior of $\log M_{n}$ as $n \rightarrow \infty$ have been studied independently by a number of authors including [9], 7], 14], 5]. Also, two versions of $\log M_{n}$ with an additional dependence between the sums $\sum_{j=1}^{n} V_{i, j}, i=1, \ldots, N_{n}$, have been studied in [13] (in the context of the Erdös-Renyi law of large numbers), and in [3, Chapter 3] (the maximal degree of a vertex in a random graph). Another related model is the maximum of the branching random walk; see, e.g., [14]. If the random variable $V$ is non-lattice with finite exponential moments, then $\log M_{n}$ 
has limiting Gumbel extreme-value distribution function $e^{-e^{-x}}$. In the lattice case, the convergence to the Gumbel limit breaks down and instead, a family of discrete analogues of the Gumbel distribution appears as the set of the weak accumulation points.

The rest of the paper is devoted to the proof of Theorem 1. Our approach (which follows the idea used in [2] and [5]) is to view $Z_{n}$ as a row sum in a triangular array with independent rows and to apply the classical theory of convergence to infinitely divisible distributions. This results in a number of conditions on the truncated exponential moments which need to be verified. The verification is done using the precise large deviation theorems due to Bahadur and Ranga Rao [1] and Petrov [16].

\section{Proof of Theorem 1 and Corollary 1}

2.1. Method of the proof. Recall that $\left\{V_{i, j} ;(i, j) \in \mathbb{N}^{2}\right\}$ and $\left\{V_{j} ; j \in \mathbb{N}\right\}$ are independent copies of a random variable $V$ satisfying (6). For every $n \in \mathbb{N}$, let $W_{1, n}, \ldots, W_{N_{n}, n}$ and $W_{n}$ be i.i.d. random variables defined by

$$
W_{i, n}=e^{\sum_{j=1}^{n} V_{i, j}-b_{n}}, \quad i=1, \ldots, N_{n} ; \quad W_{n}=e^{\sum_{j=1}^{n} V_{j}-b_{n}} .
$$

With this notation, Eqn. (12) of Theorem 1 is equivalent to the following statement:

$$
\sum_{i=1}^{N_{n_{k}}} W_{i, n_{k}}-B_{n_{k}}^{-1} A_{n_{k}} \stackrel{\mathrm{w}}{\rightarrow} \mathcal{F}_{\alpha, \Delta}, \quad k \rightarrow \infty .
$$

Note that $\left\{W_{i, n} ; n \in \mathbb{N}, i=1, \ldots, N_{n}\right\}$ is a triangular array of positive-valued random variables and that the variables within the same row are independent of each other. By the standard theory of convergence to infinitely divisible distributions (see, e.g., Theorem 1 on page 116 in [8]), the convergence in (18) will be established once we have verified the validity of the following three statements:

(1) For every $\tau>0$ with $\tau \notin e^{h \mathbb{Z}-\Delta}$,

$$
\lim _{k \rightarrow \infty} N_{n_{k}} \mathbb{P}\left[W_{n_{k}}>\tau\right]=\sum_{x \in e^{h \mathbb{Z}-\Delta}} x^{-\alpha} 1_{x>\tau}
$$

(2) We have

$$
\lim _{\tau \downarrow 0} \limsup _{k \rightarrow \infty} N_{n_{k}} \operatorname{Var}\left[W_{n_{k}} 1_{W_{n_{k}} \leq \tau}\right]=0 .
$$

(3) For every $\tau>0$ with $\tau \notin e^{h \mathbb{Z}-\Delta}$, the following limit exists and is finite:

$$
C_{\alpha, \Delta ; \tau}:=\lim _{k \rightarrow \infty}\left(N_{n_{k}} \mathbb{E}\left[W_{n_{k}} 1_{W_{n_{k}} \leq \tau}\right]-B_{n_{k}}^{-1} A_{n_{k}}\right) .
$$

Note that the first condition identifies the Lévy measure of the limiting distribution $\mathcal{F}_{\alpha, \Delta}$, the second condition shows that there is no Gaussian part in the limit, and the last condition identifies the shift parameter in the Lévy-Khintchine formula. The formula (13) for the characteristic function of the limiting distribution $\mathcal{F}_{\alpha, \Delta}$ follows from Eqn. (8) on page 84 of [8]. 
2.2. Facts about large deviations. We collect some facts on large deviations for sums of independent random variables needed in the sequel. Let $\left\{V_{i} ; i \in \mathbb{N}\right\}$ be i.i.d. copies of a random variable $V$ satisfying (6), and let $S_{n}=V_{1}+\ldots+V_{n}$ be their partial sums. Recall that

$$
\psi(t)=\log \mathbb{E} e^{t V}, \quad t \in \mathbb{R}, \quad I(\beta)=\sup _{t \in \mathbb{R}}(\beta t-\psi(t)), \quad \beta \in \mathbb{R} .
$$

Note that $\psi$ is infinitely differentiable, strictly convex, and $\psi(0)=0$. The function $I$ is finite, strictly convex and infinitely differentiable on the interval $\left(\beta_{-\infty}, \beta_{+\infty}\right)$, and its unique zero is $\beta_{0}$, where

$$
\beta_{-\infty}:=\lim _{t \rightarrow-\infty} \psi^{\prime}(t), \quad \beta_{0}:=\psi^{\prime}(0)=\mathbb{E} V, \quad \beta_{+\infty}:=\lim _{t \rightarrow+\infty} \psi^{\prime}(t) .
$$

If $\beta=\psi^{\prime}(\alpha)$ for some $\alpha \in \mathbb{R}$, then the supremum in (22) is attained at $t=\alpha$ and hence,

$$
I\left(\psi^{\prime}(\alpha)\right)=\alpha \psi^{\prime}(\alpha)-\psi(\alpha), \quad \alpha \in \mathbb{R} .
$$

The next lemma is standard; see, e.g., [10, Lemma 3] for the proof.

Lemma 1. For every $\alpha \in \mathbb{R}$, we have $I^{\prime}\left(\psi^{\prime}(\alpha)\right)=\alpha$.

The following theorem on the precise asymptotic behavior of large deviation probabilities for sums of i.i.d. variables of Bahadur and Ranga Rao [1], Petrov [16] (see Theorem 6 therein) will play a crucial role in the sequel. It is this theorem where the difference between the lattice and the non-lattice case comes into play.

Theorem 2. Suppose that (6) is satisfied. For $\beta \in\left(\beta_{-\infty}, \beta_{+\infty}\right)$ define $\alpha$ to be the unique solution of the equation $\psi^{\prime}(\alpha)=\beta$. Assume that the distribution of $V$ is lattice, and that Assumption 1 is fulfilled for some $h>0$.

(1) For every $\beta \in n^{-1} h \mathbb{Z}$,

$$
\mathbb{P}\left[S_{n}=n \beta\right] \sim \frac{h e^{-n I(\beta)}}{\sqrt{2 \pi \psi^{\prime \prime}(\alpha) n}}, \quad n \rightarrow \infty .
$$

(2) For every compact set $K \subset\left(\beta_{0}, \beta_{+\infty}\right)$, the following holds uniformly in $\beta \in n^{-1} h \mathbb{Z} \cap K$ :

$$
\mathbb{P}\left[S_{n} \geq n \beta\right] \sim \frac{h e^{-n I(\beta)}}{\left(1-e^{-\alpha h}\right) \sqrt{2 \pi \psi^{\prime \prime}(\alpha) n}}, \quad n \rightarrow \infty .
$$

(3) For every compact set $K \subset\left(\beta_{-\infty}, \beta_{0}\right)$, the following holds uniformly in $\beta \in n^{-1} h \mathbb{Z} \cap K:$

$$
\mathbb{P}\left[S_{n} \leq n \beta\right] \sim \frac{h e^{-n I(\beta)}}{\left(1-e^{\alpha h}\right) \sqrt{2 \pi \psi^{\prime \prime}(\alpha) n}}, \quad n \rightarrow \infty .
$$

In our proofs, we will several times use an exponential change of measure. Given $t_{0} \in \mathbb{R}$, we define $\tilde{V}$ (dependent on $t_{0}$ ) to be a random variable with density

$$
\mathbb{P}[\tilde{V}=d x]=e^{t_{0} x-\psi\left(t_{0}\right)} \mathbb{P}[V=d x] .
$$

Note that the right-hand side is a probability measure since $\mathbb{E}\left[e^{t_{0} V-\psi\left(t_{0}\right)}\right]=1$. 
Lemma 2. The Laplace transform $\tilde{\psi}$ and the information function $\tilde{I}$ corresponding to $\tilde{V}$ are given by

$$
\begin{aligned}
& \tilde{\psi}(t)=\psi\left(t+t_{0}\right)-\psi\left(t_{0}\right), \quad t \in \mathbb{R}, \\
& \tilde{I}(\beta)=I(\beta)+\psi\left(t_{0}\right)-t_{0} \beta, \quad \beta \in\left(\beta_{-\infty}, \beta_{+\infty}\right) .
\end{aligned}
$$

Proof. The formula for $\tilde{\psi}$ follows immediately from (28). To prove the formula for $\tilde{I}$, note that by (29),

$$
\tilde{I}(\beta)=\sup _{t \in \mathbb{R}}(\beta t-\tilde{\psi}(t))=\sup _{t \in \mathbb{R}}\left(\beta\left(t+t_{0}\right)-\psi\left(t+t_{0}\right)\right)+\psi\left(t_{0}\right)-t_{0} \beta .
$$

Since the supremum on the right-hand side equals $I(\beta)$, Eqn. (30) follows.

Let $\left\{\tilde{V}_{i} ; i \in \mathbb{N}\right\}$ be independent copies of $\tilde{V}$ and denote by $\tilde{S}_{n}=\tilde{V}_{1}+\ldots+\tilde{V}_{n}$ their partial sums. By computing the Laplace transforms one obtains immediately that

$$
\mathbb{P}\left[\tilde{S}_{n}=d x\right]=e^{t_{0} x-\psi\left(t_{0}\right) n} \mathbb{P}\left[S_{n}=d x\right] .
$$

\subsection{An auxiliary lemma.}

Lemma 3. Let the assumptions of Theorem 1 be fulfilled and let $\left\{x_{n}\right\}_{n \in \mathbb{N}}$ be a sequence with $\lim _{n \rightarrow \infty} x_{n}=x$. Then,

$$
n I\left(\frac{b_{n}+x_{n}}{n}\right)=\log \left(\frac{N_{n} h}{\sqrt{2 \pi \psi^{\prime \prime}(\alpha) n}}\right)+\alpha x+o(1), \quad n \rightarrow \infty .
$$

Proof. Recall from (10) that $b_{n}=n I^{-1}\left(c_{n}\right)$, where

$$
c_{n}=\frac{1}{n} \log \left(\frac{N_{n} h}{\sqrt{2 \pi \psi^{\prime \prime}(\alpha) n}}\right) .
$$

It follows from (4) that $\lim _{n \rightarrow \infty} c_{n}=\lambda$ and hence, $\lim _{n \rightarrow \infty} I^{-1}\left(c_{n}\right)=\psi^{\prime}(\alpha)$. [Recall that $I\left(\psi^{\prime}(\alpha)\right)=\lambda$ by (24)]. By Taylor's expansion of the function $I$ around the point $I^{-1}\left(c_{n}\right)$, we have

$$
\begin{aligned}
I\left(\frac{b_{n}+x_{n}}{n}\right) & =I\left(I^{-1}\left(c_{n}\right)+\frac{x_{n}}{n}\right) \\
& =c_{n}+I^{\prime}\left(I^{-1}\left(c_{n}\right)\right) \cdot \frac{x_{n}}{n}+o\left(\frac{1}{n}\right), \quad n \rightarrow \infty .
\end{aligned}
$$

By the continuity of $I^{\prime}$ and Lemma 1, we have

$$
\lim _{n \rightarrow \infty} I^{\prime}\left(I^{-1}\left(c_{n}\right)\right) x_{n}=I^{\prime}\left(\psi^{\prime}(\alpha)\right) x=\alpha x .
$$

Inserting this into (34) completes the proof of the lemma.

2.4. Proof of (19). Recalling that $W_{n}=e^{S_{n}-b_{n}}$ and using the fact that $S_{n}$ takes values in $h \mathbb{Z}$, we have

$$
\mathbb{P}\left[W_{n}>\tau\right]=\mathbb{P}\left[S_{n}>b_{n}+\log \tau\right]=\mathbb{P}\left[S_{n}>\left[b_{n}+\log \tau\right]_{h}\right] .
$$

Note that by (10) and (4),

$$
\lim _{n \rightarrow \infty} \frac{1}{n}\left[b_{n}+\log \tau\right]_{h}=I^{-1}(\lambda)=\psi^{\prime}(\alpha)>\psi^{\prime}(0)=\mathbb{E} V .
$$


By Theorem 2, Parts 1 and 2,

$$
\begin{aligned}
& \mathbb{P}\left[W_{n}>\tau\right] \\
& \sim \frac{h e^{-\alpha h}}{\left(1-e^{-\alpha h}\right) \sqrt{2 \pi \psi^{\prime \prime}(\alpha) n}} \exp \left\{-n I\left(\frac{\left[b_{n}+\log \tau\right] h}{n}\right)\right\}, \quad n \rightarrow \infty .
\end{aligned}
$$

By the assumption (11) of Theorem $1 \lim _{k \rightarrow \infty}\left\{b_{n_{k}}\right\}_{h}=\Delta$. Recall also that $\log \tau \notin$ $h \mathbb{Z}-\Delta$. Thus,

$$
\Theta_{\Delta ; \tau}:=\lim _{k \rightarrow \infty}\left[b_{n_{k}}+\log \tau\right]_{h}-b_{n_{k}}=[\Delta+\log \tau]_{h}-\Delta .
$$

Restricting Lemma 3 to the subsequence $\left\{n_{k}\right\}_{k \in \mathbb{N}}$, we obtain

$$
n_{k} I\left(\frac{\left[b_{n_{k}}+\log \tau\right]_{h}}{n_{k}}\right)=\log \left(\frac{N_{n_{k}} h}{\sqrt{2 \pi \psi^{\prime \prime}(\alpha) n_{k}}}\right)+\alpha \Theta_{\Delta ; \tau}+o(1), \quad k \rightarrow \infty .
$$

Applying (36) to the right-hand side of (35), we obtain

$$
\lim _{k \rightarrow \infty} N_{n_{k}} \mathbb{P}\left[W_{n_{k}}>\tau\right]=\frac{e^{-\alpha\left(\Theta_{\Delta ; \tau}+h\right)}}{1-e^{-\alpha h}} .
$$

To see that (37) is equivalent to (19), note that

$$
\sum_{x \in e^{h \mathbb{Z}-\Delta}} x^{-\alpha} 1_{x>\tau}=\sum_{k=h^{-1} \cdot[\Delta+\log \tau]_{h}+1}^{\infty} e^{-\alpha(h k-\Delta)}=\frac{e^{-\alpha\left(\Theta_{\Delta ; \tau}+h\right)}}{1-e^{-\alpha h}} .
$$

2.5. Proof of (20). Since the variance of a random variable is not greater than the second moment, it suffices to show that

$$
\lim _{\tau \downarrow 0} \limsup _{n \rightarrow \infty} N_{n} \mathbb{E}\left[W_{n}^{2} 1_{W_{n} \leq \tau}\right]=0 .
$$

To estimate the truncated moment $\mathbb{E}\left[W_{n}^{2} 1_{W_{n} \leq \tau}\right]$, we will use an exponential change of measure argument. Let $\tilde{V}$ and $\tilde{S}_{n}$ be defined as in (28) and (31) with $t_{0}=2$. By (17) and (31), we have

$$
\begin{aligned}
N_{n} \mathbb{E}\left[W_{n}^{2} 1_{W_{n} \leq \tau}\right] & =N_{n} e^{-2 b_{n}} \mathbb{E}\left[e^{2 S_{n}} 1_{S_{n} \leq b_{n}+\log \tau}\right] \\
& =N_{n} e^{\psi(2) n} e^{-2 b_{n}} \mathbb{P}\left[\tilde{S}_{n} \leq b_{n}+\log \tau\right] \\
& =N_{n} e^{\psi(2) n} e^{-2 b_{n}} \mathbb{P}\left[\tilde{S}_{n} \leq\left[b_{n}+\log \tau\right]_{h}\right] .
\end{aligned}
$$

Note that by (10) and (4), we have

$$
\lim _{n \rightarrow \infty} \frac{1}{n}\left[b_{n}+\log \tau\right]_{h}=I^{-1}(\lambda)=\psi^{\prime}(\alpha)<\psi^{\prime}(2)=\mathbb{E} \tilde{V}
$$

Let $\tau<1$ be fixed and denote by $C_{1}, C_{2}, \ldots$ constants not depending on $\tau$. By Part 3 of Theorem 2,

$$
\mathbb{P}\left[\tilde{S}_{n} \leq\left[b_{n}+\log \tau\right]_{h}\right] \sim \frac{C_{1}}{\sqrt{n}} \exp \left\{-n \tilde{I}\left(\frac{\left[b_{n}+\log \tau\right]_{h}}{n}\right)\right\}, \quad n \rightarrow \infty,
$$

where $\tilde{I}$ is the information function corresponding to $\tilde{S}_{n}$. Let $\varepsilon \in(0,2-\alpha)$. Note that by Lemma 1, $\lim _{n \rightarrow \infty} I^{\prime}\left(b_{n} / n\right)=I^{\prime}\left(\psi^{\prime}(\alpha)\right)=\alpha$. By the convexity of $I$, we have for sufficiently large $n$,

$$
n I\left(\frac{b_{n}+\log \tau}{n}\right) \geq n I\left(\frac{b_{n}}{n}\right)+I^{\prime}\left(\frac{b_{n}}{n}\right) \log \tau \geq n I\left(\frac{b_{n}}{n}\right)+(\alpha+\varepsilon) \log \tau .
$$


Note that $\tilde{I}$ is decreasing on $\left(\beta_{-\infty}, \psi^{\prime}(2)\right)$. By Lemma 2 and Eqns. (41), (10), we have

$$
\begin{aligned}
n \tilde{I}\left(\frac{\left[b_{n}+\log \tau\right]_{h}}{n}\right) & \geq n \tilde{I}\left(\frac{b_{n}+\log \tau}{n}\right) \\
& =n I\left(\frac{b_{n}+\log \tau}{n}\right)+\psi(2) n-2\left(b_{n}+\log \tau\right) \\
& \geq \log \left(\frac{N_{n}}{\sqrt{n}}\right)+(\alpha+\varepsilon-2) \log \tau+\psi(2) n-2 b_{n}-C_{2} .
\end{aligned}
$$

Bringing (39), (40), (42) together, we obtain

$$
\limsup _{n \rightarrow \infty} N_{n} \mathbb{E}\left[W_{n}^{2} 1_{W_{n} \leq \tau}\right] \leq C_{3} \tau^{2-\alpha-\varepsilon} .
$$

Letting $\tau \downarrow 0$ and recalling that $\alpha+\varepsilon \leq 2$ yields (38).

2.6. Proof of (21). Let $\tilde{V}$ and $\tilde{S}_{n}$ be the exponential twists of $V$ and $S_{n}$ defined as in (28) and (31) with $t_{0}=1$, i.e.,

$$
\mathbb{P}[\tilde{V}=d x]=e^{x-\psi(1)} \mathbb{P}[V=d x], \quad \mathbb{P}\left[\tilde{S}_{n}=d x\right]=e^{x-\psi(1) n} \mathbb{P}\left[S_{n}=d x\right] .
$$

It follows from (11) that

$$
\Theta_{\Delta ; \tau}:=\lim _{k \rightarrow \infty} \theta_{n_{k}}=[\Delta+\log \tau]_{h}-\Delta, \text { where } \theta_{n}=\left[b_{n}+\log \tau\right]_{h}-b_{n} .
$$

Consider first the case $\alpha \in(0,1)$. Note that in this case, $A_{n}=0$ by (91). By (43), we have

$$
\begin{aligned}
N_{n} \mathbb{E}\left[W_{n} 1_{W_{n} \leq \tau}\right]-B_{n}^{-1} A_{n} & =N_{n} e^{-b_{n}} \mathbb{E}\left[e^{S_{n}} 1_{S_{n} \leq b_{n}+\log \tau}\right] \\
& =N_{n} e^{\psi(1) n} e^{-b_{n}} \mathbb{P}\left[\tilde{S}_{n} \leq b_{n}+\log \tau\right] \\
& =N_{n} e^{\psi(1) n} e^{-b_{n}} \mathbb{P}\left[\tilde{S}_{n} \leq\left[b_{n}+\log \tau\right]_{h}\right] .
\end{aligned}
$$

By (10), (4), and the assumption $\alpha \in(0,1)$,

$$
\lim _{n \rightarrow \infty} \frac{1}{n}\left[b_{n}+\log \tau\right]_{h}=I^{-1}(\lambda)=\psi^{\prime}(\alpha)<\psi^{\prime}(1)=\mathbb{E} \tilde{V}
$$

Note that by Lemma 2, $\psi^{\prime}(\alpha)=\tilde{\psi}^{\prime}(\alpha-1)$. By Part 3 of Theorem 2

$$
\begin{aligned}
& \mathbb{P}\left[\tilde{S}_{n} \leq\left[b_{n}+\log \tau\right]_{h}\right] \\
& \sim \frac{h}{\left(1-e^{(\alpha-1) h}\right) \sqrt{2 \pi \psi^{\prime \prime}(\alpha) n}} \exp \left\{-n \tilde{I}\left(\frac{\left[b_{n}+\log \tau\right]_{h}}{n}\right)\right\}, \quad n \rightarrow \infty .
\end{aligned}
$$

By (44), Lemma 2, and Lemma 3, we have

$$
\begin{aligned}
& n_{k} \tilde{I}\left(\frac{\left[b_{n_{k}}+\log \tau\right]_{h}}{n_{k}}\right) \\
& =n_{k} \tilde{I}\left(\frac{b_{n_{k}}+\theta_{n_{k}}}{n_{k}}\right) \\
& =n_{k} I\left(\frac{b_{n_{k}}+\theta_{n_{k}}}{n_{k}}\right)+\psi(1) n_{k}-\left(b_{n_{k}}+\theta_{n_{k}}\right) \\
& =\log \left(\frac{N_{n_{k}} h}{\sqrt{2 \pi \psi^{\prime \prime}(\alpha) n_{k}}}\right)+(\alpha-1) \Theta_{\Delta ; \tau}+\psi(1) n_{k}-b_{n_{k}}+o(1), \quad k \rightarrow \infty
\end{aligned}
$$


Bringing (45), (46), (47) together, we obtain

$$
C_{\alpha, \Delta ; \tau}:=\lim _{k \rightarrow \infty} N_{n_{k}} \mathbb{E}\left[W_{n_{k}} 1_{W_{n_{k}} \leq \tau}\right]=\frac{e^{-(\alpha-1) \Theta_{\Delta ; \tau}}}{1-e^{(\alpha-1) h}} .
$$

Let us consider the case $\alpha=1$. We have $B_{n}^{-1} A_{n}=N_{n} \mathbb{E}\left[W_{n} 1_{W_{n}<1}\right]$ by (9), (10). Assume for concreteness that $\tau>1$. It follows from (43) that

$$
\begin{aligned}
N_{n} \mathbb{E}\left[W_{n} 1_{W_{n} \leq \tau}\right]-B_{n}^{-1} A_{n} & =N_{n} \mathbb{E}\left[W_{n} 1_{W_{n} \in[1, \tau]}\right] \\
& =N_{n} e^{-b_{n}} \mathbb{E}\left[e^{S_{n}} 1_{b_{n}} \leq S_{n} \leq b_{n}+\log \tau\right] \\
& =N_{n} e^{\psi(1) n} e^{-b_{n}} \mathbb{P}\left[b_{n} \leq \tilde{S}_{n} \leq b_{n}+\log \tau\right] .
\end{aligned}
$$

This may be written as

$$
N_{n} \mathbb{E}\left[W_{n} 1_{W_{n} \leq \tau}\right]-B_{n}^{-1} A_{n}=N_{n} e^{\psi(1) n} e^{-b_{n}} \sum_{\substack{0 \leq j \leq \log \tau \\ j \in h \mathbb{Z}-b_{n}}} \mathbb{P}\left[\tilde{S}_{n}=b_{n}+j\right] .
$$

By Part 1 of Theorem 2, we have

$$
\mathbb{P}\left[\tilde{S}_{n}=b_{n}+j\right] \sim \frac{h}{\sqrt{2 \pi \psi^{\prime \prime}(1) n}} \exp \left\{-n \tilde{I}\left(\frac{b_{n}+j}{n}\right)\right\}, \quad n \rightarrow \infty .
$$

By Lemma 2 and Lemma 3 ,

$$
\begin{aligned}
& n_{k} \tilde{I}\left(\frac{b_{n_{k}}+j}{n_{k}}\right) \\
& =n_{k} I\left(\frac{b_{n_{k}}+j}{n_{k}}\right)+\psi(1) n_{k}-\left(b_{n_{k}}+j\right) \\
& =\log \left(\frac{N_{n_{k}} h}{\sqrt{2 \pi \psi^{\prime \prime}(\alpha) n_{k}}}\right)+\psi(1) n_{k}-b_{n_{k}}+o(1), \quad k \rightarrow \infty .
\end{aligned}
$$

Note that the right-hand side does not depend on $j$. It follows from (11) that for sufficiently large $n$, the number of summands on the right-hand side of (49) is equal to $h^{-1} \cdot[\log \tau+\Delta]_{h}$. Using (49), (50), (51), we obtain

$$
C_{1, \Delta ; \tau}:=\lim _{k \rightarrow \infty}\left(N_{n_{k}} \mathbb{E}\left[W_{n_{k}} 1_{W_{n_{k}} \leq \tau}\right]-B_{n_{k}}^{-1} A_{n_{k}}\right)=\frac{1}{h} \cdot[\log \tau+\Delta]_{h} .
$$

Finally, let us consider the case $\alpha \in(1,2)$. First note that by (9), (10), we have $B_{n}^{-1} A_{n}=N_{n} \mathbb{E}\left[W_{n}\right]$. By (43),

$$
\begin{aligned}
N_{n} \mathbb{E}\left[W_{n} 1_{W_{n} \leq \tau}\right]-B_{n}^{-1} A_{n} & =-N_{n} e^{-b_{n}} \mathbb{E}\left[e^{S_{n}} 1_{S_{n}>b_{n}+\log \tau}\right] \\
& =-N_{n} e^{\psi(1) n} e^{-b_{n}} \mathbb{P}\left[\tilde{S}_{n}>b_{n}+\log \tau\right] \\
& =-N_{n} e^{\psi(1) n} e^{-b_{n}} \mathbb{P}\left[\tilde{S}_{n}>\left[b_{n}+\log \tau\right]_{h}\right] .
\end{aligned}
$$

Note that by (10), (4), and the assumption $\alpha \in(1,2)$, we have

$$
\lim _{n \rightarrow \infty} \frac{1}{n}\left[b_{n}+\log \tau\right]_{h}=I^{-1}(\lambda)=\psi^{\prime}(\alpha)>\psi^{\prime}(1)=\mathbb{E} \tilde{V} .
$$

By Lemma 2, $\psi^{\prime}(\alpha)=\tilde{\psi}^{\prime}(\alpha-1)$. By Parts 1 and 2 of Theorem 2 ,

$$
\begin{aligned}
& \mathbb{P}\left[\tilde{S}_{n}>\left[b_{n}+\log \tau\right]_{h}\right] \\
& \sim \frac{h}{\left(e^{(\alpha-1) h}-1\right) \sqrt{2 \pi \psi^{\prime \prime}(\alpha) n}} \exp \left\{-n \tilde{I}\left(\frac{\left[b_{n}+\log \tau\right]_{h}}{n}\right)\right\}, \quad n \rightarrow \infty .
\end{aligned}
$$


Recall that $\Theta_{\Delta ; \tau}$ and $\theta_{n}$ are given by (44). By Lemma 2 and Lemma 3 , we have

$$
\begin{aligned}
& n_{k} \tilde{I}\left(\frac{\left[b_{n_{k}}+\log \tau\right]_{h}}{n_{k}}\right) \\
& =n_{k} \tilde{I}\left(\frac{b_{n_{k}}+\theta_{n_{k}}}{n_{k}}\right) \\
& =n_{k} I\left(\frac{b_{n_{k}}+\theta_{n_{k}}}{n_{k}}\right)+\psi(1) n_{k}-\left(b_{n_{k}}+\theta_{n_{k}}\right) \\
& =\log \left(\frac{N_{n_{k}} h}{\sqrt{2 \pi \psi^{\prime \prime}(\alpha) n_{k}}}\right)+(\alpha-1) \Theta_{\Delta ; \tau}+\psi(1) n_{k}-b_{n_{k}}+o(1), k \rightarrow \infty .
\end{aligned}
$$

Bringing (53), (54), (55) together, we obtain

$$
C_{\alpha, \Delta ; \tau}=\lim _{k \rightarrow \infty} N_{n_{k}} \mathbb{E}\left[W_{n_{k}} 1_{W_{n_{k}} \leq \tau}\right]-B_{n_{k}}^{-1} A_{n_{k}}=\frac{e^{-(\alpha-1) \Theta_{\Delta ; \tau}}}{1-e^{(\alpha-1) h}} .
$$

This completes the proof of (21) and the proof of Theorem 1.

2.7. Proof of Corollary 1. The relative compactness of the sequence (14) (as well as the description of its weak cluster set) follow from the fact that from every increasing integer sequence we can extract a subsequence $n_{k}$ satisfying (11) with some $\Delta \in[0, h]$ and then apply Theorem 1 .

Let us prove that for every fixed $\alpha \in(0,2)$, the set $\left\{\mathcal{F}_{\alpha, \Delta} ; \Delta \in[0, h]\right\}$ is homeomorphic to a circle. Recall from (13) that the logarithm of the characteristic function of $\mathcal{F}_{\alpha, \Delta}$ is given by

$$
\log \phi_{\alpha, \Delta}(u)=i C_{\alpha, \Delta ; \tau} u+\sum_{x \in e^{h \mathbb{Z}-\Delta}}\left(e^{i u x}-1-i u x 1_{x<\tau}\right) x^{-\alpha}, \quad u \in \mathbb{R},
$$

where $\tau>0$ is arbitrary with $\tau \notin e^{h \mathbb{Z}-\Delta}$. It follows from (44) that $\Theta_{0 ; \tau}=\Theta_{h ; \tau}$. Then, Eqns. (48), (52), (56) imply that $C_{\alpha, 0 ; \tau}=C_{\alpha, h ; \tau}$. Trivially, we have $e^{h \mathbb{Z}}=$ $e^{h \mathbb{Z}-h}$. By (57), it follows from these facts that $\mathcal{F}_{\alpha, 0}=\mathcal{F}_{\alpha, h}$. On the other hand, the Lévy measure of $\mathcal{F}_{\alpha, \Delta}$ is given by $\sum_{x \in e^{h \mathbb{Z}-\Delta}} x^{-\alpha} \delta_{x}$, which implies that the distributions $\mathcal{F}_{\alpha, \Delta}, \Delta \in[0, h)$, are different.

To complete the proof, we need to show that $\mathcal{F}_{\alpha, \Delta}$ depends continuously (in the weak topology) on $\Delta$. Take some $\Delta_{0} \in[0, h]$ and choose $\tau>0$ such that $\tau \notin e^{h \mathbb{Z}-\Delta_{0}}$. It is easily seen from (44) and (48), (52), (56) that $C_{\alpha, \Delta ; \tau}$ is a continuous function of $\Delta$ in a neighborhood of $\Delta_{0}$. It follows from (57) that $\lim _{\Delta \rightarrow \Delta_{0}} \phi_{\alpha, \Delta}(u)=\phi_{\alpha, \Delta_{0}}(u)$ for every $u \in \mathbb{R}$. By the Lévy continuity theorem, this implies that $\mathcal{F}_{\alpha, \Delta}$ is a continuous function of $\Delta$. This completes the proof of Corollary 1 .

\section{ACKNOWLEDGEMENTS}

The author is grateful to Leonid Bogachev for pointing out the references [5] and [12].

\section{REFERENCES}

[1] R. Bahadur and R. Ranga Rao. On deviations of the sample mean. Ann. Math. Stat., 31:1015-1027, 1960.

[2] G. Ben Arous, L. Bogachev, and S. Molchanov. Limit theorems for sums of random exponentials. Probab. Theory Relat. Fields, 132(4):579-612, 2005. 
[3] B. Bollobás. Random graphs, volume 73 of Cambridge Studies in Advanced Mathematics. Cambridge University Press, Cambridge, second edition, 2001.

[4] A. Bovier, I. Kurkova, and M. Löwe. Fluctuations of the free energy in the REM and the $p$-spin SK models. Ann. Probab., 30(2):605-651, 2002.

[5] M. Cranston and S. Molchanov. Limit laws for sums of products of exponentials of iid random variables. Isr. J. Math., 148:115-136, 2005.

[6] S. Csörgö and R. Dodunekova. Limit theorems for the Petersburg game. In Sums, trimmed sums and extremes, volume 23 of Progr. Probab., pages 285315. Birkhäuser Boston, Boston, MA, 1991.

[7] R. Durrett. Maxima of branching random walks vs. independent random walks. Stochastic Process. Appl., 9(2):117-135, 1979.

[8] B.V. Gnedenko and A.N. Kolmogorov. Limit distributions for sums of independent random variables. Cambridge: Addison-Wesley Publishing Company, 1954.

[9] G. Ivchenko. Variational series for a scheme of summing independent variables. Theory of Probab. Appl., 18:531-545, 1973.

[10] Z. Kabluchko. Limiting distributions for sums of independent random products. Not published. Available at http://arxiv.org/abs/0904.4127, 2009.

[11] Z. Kabluchko. Functional limit theorems for sums of independent geometric Lévy processes. Submitted, 2009. Available at http://arxiv.org/abs/0911.4139.

[12] O. Khorunzhiy. Limit theorem for sums of products of random variables. Markov Process. Related Fields, 9(4):675-686, 2003.

[13] J. Komlos and G. Tusnády. On sequences of "pure heads". Ann. Probab., 3: 608-617, 1975.

[14] M. Lifshits. Some limit theorems on binary trees. Notes.

[15] A. Martin-Löf. A limit theorem which clarifies the "Petersburg paradox". J. Appl. Probab., 22(3):634-643, 1985.

[16] V. Petrov. On the probabilities of large deviations for sums of independent random variables. Theor. Probab. Appl., 10:287-298, 1965.

[17] R. Pillai. Semi stable laws as limit distributions. Ann. Math. Statist., 42(2): 780-783, 1971.

[18] K.-I. Sato. Lévy processes and infinitely divisible distributions. Cambridge Studies in Advanced Mathematics. 68. Cambridge University Press., 1999. 